\title{
Increased diversification rates follow shifts to bisexuality in
}

\section{liverworts}

\author{
Benjamin Laenen $^{1,2}$, Antonin Machac ${ }^{3,4,5}$, S. Robbert Gradstein ${ }^{6}$, Blanka Shaw ${ }^{7}$, Jairo Patiño ${ }^{2}$, \\ Aurélie Désamoré $^{2,8}$, Bernard Goffinet ${ }^{9}$, Cymon J. Cox ${ }^{10}$, A. Jonathan Shaw ${ }^{7}$ and Alain Vanderpoorten ${ }^{2 *}$
}

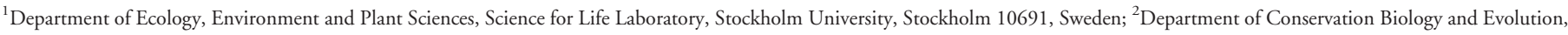
Institute of Botany, University of Liège, Liège 4000, Belgium; ${ }^{3}$ Center for Macroecology, Evolution, and Climate, Natural History Museum of Denmark, Universitetsparken 15 , DK 2100

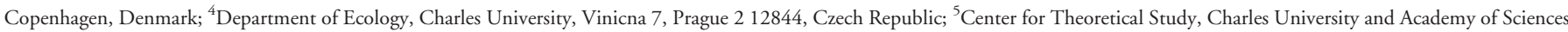
of the Czech Republic, Jilska 1, Prague 1 11000, Czech Republic; ${ }^{6}$ Département Systématique et Evolution, Muséum National d'Histoire Naturelle, Paris 75005 , France; ${ }^{7}$ Department of

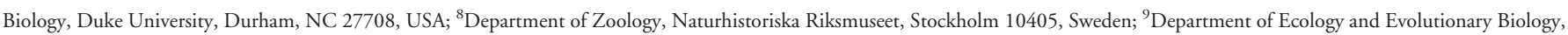
University of Connecticut, Storrs, CT 06269, USA; ${ }^{10}$ Centro de Ciências do Mar (CCMAR), Universidade do Algarve, Campus de Gambelas, Faro 8005-139, Portugal

Author for correspondence: Benjamin Laenen

Tel: +3243663842

Email: benjaminlaenen@hotmail.com

Received: 19 October 2015

Accepted: 1 December 2015

New Phytologist (2016) 210: 1121-1129 doi: 10.1111/nph.13835

Key words: Baker's law, dispersal, diversification, hidden state speciation and extinction analysis (HiSSE), liverworts, sexual systems.

\section{Summary}

- Shifts in sexual systems are one of the key drivers of species diversification. In contrast to angiosperms, unisexuality prevails in bryophytes. Here, we test the hypotheses that bisexuality evolved from an ancestral unisexual condition and is a key innovation in liverworts.

- We investigate whether shifts in sexual systems influence diversification using hidden state speciation and extinction analysis (HiSSE). This new method compares the effects of the variable of interest to the best-fitting latent variable, yielding robust and conservative tests.

- We find that the transitions in sexual systems are significantly biased toward unisexuality, even though bisexuality is coupled with increased diversification. Sexual systems are strongly conserved deep within the liverwort tree but become much more labile toward the present.

- Bisexuality appears to be a key innovation in liverworts. Its effects on diversification are presumably mediated by the interplay of high fertilization rates, massive spore production and long-distance dispersal, which may separately or together have facilitated liverwort speciation, suppressed their extinction, or both. Importantly, shifts in liverwort sexual systems have the opposite effect when compared to angiosperms, leading to contrasting diversification patterns between the two groups. The high prevalence of unisexuality among liverworts suggests, however, a strong selection for sexual dimorphism.

\section{Introduction}

Plant sexual systems are highly diverse and have long stimulated research by ecologists, phylogeneticists, and population geneticists (Cheptou \& Schoen, 2007). In angiosperms, as many as 5000 independent origins of unisexuality (i.e. the production of male and female gametangia by different individuals) may have occurred (Renner, 2015) and shifts between sexual systems appear to be a major driver of species diversification (Barrett, 2013). In seed plants, unisexual species might appear to suffer a competitive disadvantage compared with bisexual species (i.e. species wherein both male and female gametangia are produced by the same individual) because only half of the individuals in a unisexual population are seed-bearing. Thus, maintenance of unisexuality in the presence of bisexual competitors requires a substantial increase in relative fitness of unisexual individuals and/or a large dispersal advantage of unisexual seeds (Heilbuth

*These authors contributed equally to this work. et al., 2001). In addition, self-compatible bisexual species are more likely to establish new colonies following long-distance dispersal than unisexual or self-incompatible ones (Bakers's Law; Baker, 1955, 1967), potentially promoting allopatric speciation (Heilbuth, 2000). Comparing bisexual and unisexual sisterclades, Heilbuth (2000) found, indeed, a significantly lower species richness in unisexual clades, leading to the conclusion that the low proportion of unisexual species (c. 6\%) in the world angiosperm flora might be explained by lower speciation and/or higher extinction rates (Heilbuth et al., 2001; Vamosi \& Otto, 2002; Vamosi \& Vamosi, 2004).

In mosses, McDaniel et al. (2013), using both sister-clade comparisons and Binary State Speciation and Extinction analysis (BiSSE, Maddison et al., 2007), found a similarly slight trend towards increased diversification in bisexual moss lineages. The null expectation of equal species richness in sister-clade comparisons could, however, be wrong in the case of a derived trait occurring independently from speciation (Käfer \& Mousset, 2014). Moreover, BiSSE analyses were recently shown to 
improperly account for phylogenetic independence in the data (Maddison \& FitzJohn, 2015) and exhibit high levels of false positive tests, leading Rabosky \& Goldberg (2015) to suggest that many trait-driven diversifications reported in the literature may not be real. Most recently, Beaulieu \& O'Meara (2015) further suggested that it is still unclear whether BiSSE uncovers actual important drivers of diversification, or whether the model simply points to more complex patterns involving many unmeasured and co-distributed factors. These concerns cast doubt on previous analyses identifying bisexuality as a diversification driver in mosses (McDaniel et al., 2013) and angiosperms (Heilbuth et al., 2001). Indeed, Käfer etal. (2014) re-analyzed the largest angiosperm phylogeny available to date and, in contrast to Heilbuth (2000), concluded that unisexuality is associated with higher diversification rates. This pattern is supported by studies showing that the apparent short-term advantages of selffertilization are offset by strong species selection, which favors obligate outcrossing (Goldberg et al., 2010; Wright \& Barrett, 2010). In particular, De Vos et al. (2014) found, in their study on the evolution of heterostyly in primroses, that outcrossing species do not exhibit higher speciation rates than self-pollinating ones. Instead, the higher diversification rates of outcrossing species, as compared to self-compatible ones, was explained by lower extinction rates presumably because these species avoid the negative genetic effects of self-fertilization, including reduced genetic diversity and lower rates of effective recombination, leading to relaxed purifying selection and decreased opportunities for adaptive mutations to become fixed (Wright \& Barrett, 2010).

Bryophytes are especially well-suited to investigate the impact of variation in sexual systems on evolutionary success because they exhibit an unparalleled lability in this character (see McDaniel etal., 2013, for mosses and Villarreal \& Renner, 2013, for hornworts). In contrast to angiosperms, unisexuality, which characterizes $c .70 \%$ of liverworts (Supporting Information Table S1) and 60\% of mosses (Patiño et al., 2013), has traditionally been perceived as the ancestral state in these two lineages (Longton \& Schuster, 1983). In bryophytes, sexual reproduction requires water for sperm to swim to eggs. In bisexual species, this functional constraint is virtually lacking. Indeed, although it should not be assumed that bisexual species are selfing simply because the male and female gametangia are simultaneously mature, and although one case of self-incompatibility was recently reported (Stark \& Brinda, 2013), bisexual bryophyte species are assumed to be capable of self-fertilization. Selfing in bisexual bryophytes is evidenced by the very high $F_{\text {is }}$ values observed in the sporophytic phase of all bisexual species investigated so far (Eppley et al., 2007; Hutsemékers et al., 2013; Johnson \& Shaw, 2015; Klips, 2015).

In contrast to bisexual species, and despite evidence for desiccation tolerance of sperm cells in mosses (Shortlidge et al., 2012), the likelihood of fertilization in unisexual species is inversely proportional to the distance between male and female plants. Antherozoids are capable of swimming as far as $1-2 \mathrm{~m}$ at a speed of $100-200 \mu \mathrm{m} \mathrm{s}^{-1}$. The greatest distance between fruiting plants and the nearest male inflorescence has been recorded at a maximum of $3.8 \mathrm{~m}$ (Longton, 1997). However, fertilization ranges are generally much shorter. Rydgren etal. (2006) found, for example, that $85 \%$ of the female shoots with sporophytes were situated within a distance of $5 \mathrm{~cm}$ from the nearest male and the longest distance was $11.6 \mathrm{~cm}$. Sexual reproduction in unisexual species may be further challenged by: the strongly biased sex ratio of their populations (Bisang etal., 2014); the spatial segregation of the sexes due to differences in niche preference between males and females (Stark \& McLetchie, 2006; Brzyski et al., 2014; but see Bisang etal., 2015); and the potential incompatibility between certain male and female genotypes in unisexual species (McLetchie, 1996). Fertilization and sporophyte production are therefore substantially higher in bisexual species (Longton, 1997). Given that spores have long been assumed to be involved in long-distance dispersal (but see Laenen et al., 2015), we expect anagenetic speciation by allopatry (i.e. the gradual evolution of a new species from a founder event), the main speciation driver in bryophytes (Patiño et al., 2014), to be higher in bisexual than in unisexual species. Therefore, we may expect that bisexuality was a key innovation, that is, a trait that significantly increases the diversification rate (see Donoghue \& Sanderson, 2015, for review).

In this study, we take liverworts as a model to address the following questions and test the following hypotheses: (1) Do shifts in sexual systems correlate with altered diversification rates? Assuming that bisexual species experience high levels of allopatric speciation, we expect that bisexuality is a key innovation, so that bisexual lineages are expected to exhibit higher diversification rates than unisexual ones. (2) What was the ancestral sexual condition in liverworts and how did it evolve? We expect that bisexuality evolved from an ancestral unisexual condition (Longton \& Schuster, 1983), with few if any reverse shifts, leading to a strong heritability of sexual systems across the liverwort phylogeny.

\section{Materials and Methods}

\section{Phylogenetic framework}

A time-scaled liverwort phylogeny was derived from the analyses of Laenen et al. (2014), which included one species for 297 out of the 382 liverwort genera currently recognized. Performing the analyses on an ultrametric tree is consistent with the notion that trait evolution is related to time, but not necessarily to the number of substitutions (Pellicer et al., 2014; but see Litsios \& Salamin, 2012, and Cusimano \& Renner, 2014). The chronogram was the maximum clade credibility tree resulting from the relaxed-clock Bayesian analysis of 297 liverwort genera sequenced for five chloroplast genes ( $a t p \mathrm{~B}, p s b \mathrm{~A}, p s b \mathrm{~T}, r b c \mathrm{~L}$ and $r p s 4)$, two mitochondrial genes ( $r p s 3$ and nad1) and the $26 \mathrm{~S}$ nuclear ribosomal RNA gene. The chronogram was calibrated with 25 fossils, using a log-normal distribution of prior probabilities of ages centered on the fossil age estimate with a standard deviation set so that its range encompasses the upper limit of the oldest inferred estimate of the origin of land plants, $815 \mathrm{Myr}$ ago (Ma) (Litsios \& Salamin, 2012; Calibration III in Laenen et al., 2014). One hundred trees were also randomly sampled from the posterior distribution of trees from the BEAST analysis of Laenen etal. 
(2014) to take phylogenetic uncertainty into account. The trees are available from TreeBASE (http://www.treebase.org) under accession ID 15950.

\section{Data collection}

In comparative phylogenetic analyses, sampling at the genus level raises the issue that 'higher-level terminals require dealing with variation among the species that make up the terminal taxa' (Wiens, 1998). As FitzJohn et al. (2009) noted, in particular, 'the BISSE [Binary State Speciation and Extinction analysis] method as formulated by Maddison et al. (2007) assumes that the phylogenetic tree is complete and fully resolved; that is, the tree must include every extant species'. It also assumes that all character state information is known. These assumptions currently restrict its applicability, as few published phylogenies are both complete to the species level and large enough to detect differential diversification. Here, the benefit of a large-scale analysis potentially increasing our ability to detect trait-dependent diversification shifts may be confounded by the severely incomplete sample of species. The extreme limitations of incomplete sampling at the species level prevented us from implementing the 'terminally unresolved tree' strategy proposed by FitzJohn et al. (2009), which cannot handle datasets with $>200$ missing species per terminal branch (FitzJohn, 2012). Several scoring strategies have been employed to account for trait polymorphism among species, including (1) sampling a single species per higher taxon, (2) coding variable taxa as missing or polymorphic and (3) coding inferred ancestral states (Wiens, 1998). The single-species selection (1) enforces a very strong constraint on that node, as the sexual condition of the sampled species may not be representative of the main sexual condition of the genus. Coding variable genera as missing (2) would lead to a substantial loss of information. Therefore, our strategy was to assign the sexual condition of each genus based on the modal condition, that is a genus was scored as unisexual or bisexual when $>75 \%$ of its constitutive species met that condition. Although 74 out of the 297 investigated genera included both unisexual and bisexual species (Table S1), this strategy led us to score genera with $50-75 \%$ of either unisexual or bisexual species ( 40 genera) as missing. To assess the impact of the loss of information caused by the scoring of those 40 variable genera as missing, we also performed the analysis using a more stringent threshold of $90 \%$, leading to the scoring of a further 24 genera as missing. Following other recent genus-level diversification analyses (Schwery et al., 2015), we then attempted to correct for sampling incompleteness (only one species per genus) by using a sampling fraction that was informed by the global proportion of unisexual and bisexual species. Thus, 205 unisexual and 58 bisexual species sampled represented $5.9 \%$ and $4.3 \%$ of the 3426 and 1339 unisexual and bisexual species, respectively (Table S1). Still, sampling at the genus level could potentially bias our assessment of the transition rates by ignoring infrageneric variation of the sexual condition in the $74(25 \%)$ polymorphic genera. In angiosperms, self-compatible species appear at the tips of the tree because of higher rates of extinction in selfcompatible lineages, as demonstrated by Goldberg et al. (2010) and De Vos etal. (2014). Sampling genera as terminal units could lead to biased sexual transition rates because of high rates of extinction of bisexual lineages, so that many transitions towards bisexuality would be hidden. Our best-fit diversification model, however, involves equal rates of extinction in unisexual and bisexual lineages (Table S1, S3). Then, we do not have any reason to believe that the proportion of bisexual lineages varies along the tree, and hence, that sampling at the species level would substantially alter our estimates of sexual transition rates.

\section{Phylogenetic signal in trait evolution}

The statistical significance of the phylogenetic signal associated with sexual systems was tested by comparing the fit of phylogenetic (chronogram) and nonphylogenetic (star-tree) models to the data (Mooers et al., 1999). For this purpose, trait evolution was modeled by estimating the instantaneous rates of transitions among all possible pairs of states by using the 'multistate' Markov model of BayesTraits v.2.0 (Pagel et al., 2004). The fit of oneor several-rate models was first determined for each character and for each tree using a likelihood ratio test (LRT). The best-fit model was then implemented to describe trait evolution on the chronogram tree and on a star-tree, the latter tree having all internal branches set to 0 and the length of each terminal branch corresponding to the total path from the root to the extant species using the R package 'geiger' (Harmon et al., 2008). The phylogenetic and nonphylogenetic models include the same number of parameters, and therefore their likelihood values were directly comparable, with a difference of two being considered statistically significant (Mooers et al., 1999; Oakley et al., 2005). However, the presence of phylogenetic signal can vary depending on the taxonomic level of the analysis because antagonistic patterns at different levels can obscure one another when the analysis of phylogenetic signal is conducted simultaneously across the entire phylogeny (Machac et al., 2011). Analyses were, therefore, conducted for each node with $>10$ operational taxonomic units and reported on the chronogram.

In order to determine the proportion of nodes for which a significant phylogenetic signal was observed by chance (Type I error), we simulated the distribution of states that would be expected for characters with contrasting levels of phylogenetic signal. The tests described above were therefore applied to randomly shuffled characters to derive the distribution of the $P$-values associated to the hierarchical LRT for random characters. To estimate the effect of phylogenetic uncertainty, the analyses were repeated on the 100 trees randomly sampled.

\section{Character associated diversification}

In order to solve the problems that BiSSE almost always incorrectly find neutral traits correlated with higher diversification (Rabosky $\&$ Goldberg, 2015) and does not properly account for phylogenetic independence in the data (Maddison \& FitzJohn, 2015), we tested the hypothesis that shifts in sexual systems correlate with shifts in diversification rates by applying hidden state speciation and extinction analyses (HiSSE; Beaulieu \& O’Meara, 2015). 
As Beaulieu \& O’Meara (2015) emphasize, rejecting a null model of constant diversification rates because it is too simple does not necessarily imply that the more complex trait-dependent diversification model implemented is correct. HiSSE circumvents this issue by implementing a null model that explicitly assumes that the evolution of a binary character is independent of the diversification process without forcing diversification process to be constant across the entire tree. Hence, the null model contains the same degree of complexity in terms of numbers of parameters to allow for comparisons among any complex model of interest, avoiding the trap of choosing a model purely on the basis of it having more parameters. HiSSE further accounts for the presence of unmeasured factors that could impact diversification rates estimated for the states of any observed trait. Therefore, HiSSE implements two null models (schematically explained in Fig. 1 of Beaulieu \& O'Meara, 2015). The first model, referred to as 'nulltwo', contains four diversification process parameters that account for trait-dependent diversification solely on the two states of an unobserved, hidden trait, so that null-two contains the same amount of complexity in terms of diversification as a BiSSE model. The second model, referred to as 'null-four', contains the same number of diversification parameters as in the general HiSSE model. We tested 22 competing models, from the null models to the complete HiSSE model. These 22 models implemented identical transition rates (q) among states of the observed and hidden character, respectively, but exhibited increasing levels of complexity depending on whether turnover rates (tau), extinction fraction (eps), or the combination thereof, were allowed to vary between unisexual and bisexual lineages. To test the hypothesis that shifts among states of the observed (sexual systems) and hidden characters might account for shifts in transition rates, these 22 models were then contrasted with another 22 models, wherein transition rates of the observed and hidden states were not constrained. In total, we fitted 44 models to each of the two datasets and selected the best-fit model using the Akaike Information Criterion corrected for sample size (AICc), using a delta AICc of 2 to select the best-fit model(s). After selection of the best-fit model, we estimated the confidence interval of each parameter (tau, eps and q) by sampling parameter values, ensuring that the difference of $\log$-likelihood $<2$ as compared to the best-fit model as implemented in the 'SupportRegion' function. Tau and eps are transformed to speciation and extinction rates using Eqn 5(a,b) from Beaulieu \& O'Meara (2015). We therefore report the results as speciation (lambda), extinction (mu) and net diversification rates (lambda $-\mathrm{mu}$ ) to allow comparison with others studies.

In order to account for phylogenetic uncertainty we fitted the best-fit model identified above on the 100 trees randomly sampled from the posterior probability distribution of Laenen et al. (2014). This model was then compared to the 'null four' model and a HiSSE model with equal turnover rates across the 100 trees.

The most likely set of ancestral sexual systems was reconstructed across nodes after having set the transition rates at their most likely value following Schluter et al.'s (1997) optimization, as implemented by the 'MarginRecon' function. To take model uncertainty into account, we used model averaging by AIC so that the contribution of each model to the final estimate was proportional to its likelihood.

\section{Results}

Regardless of the threshold used to assign a state to each tip ( $>75 \%$ and $>90 \%$ of either unisexual or bisexual species; scoring the state of 40 and 64 genera, respectively, missing out of 297), inferences of ancestral sexual condition and traits linked to diversification were very similar. This suggests that, although our analyses did not fully capture the potential impact of infrageneric variation in the 74 polymorphic genera, the results seems to be robust to the threshold employed to assign a sexual condition to each genus using a majority rule and to the loss of information caused by the scoring of the state of 40 polymorphic genera with a $50-75 \%$ proportion of uni- or bisexual species as missing data.

The best-fit model for both scoring schemes was a HiSSE model with an extinction fraction equal in unisexual and bisexual lineages, and different transition rates between unisexuality and bisexuality (model 30; Tables S2, S3) regardless of scoring scheme and exclusion of data. For the $75 \%$ majority-rule dataset, this model had an AIC weight of 96\% (Table S2). For the 90\% majority-rule dataset, the AIC weight dropped to $68 \%$. The AIC weight of a model with equal diversification rates between unisexual and bisexual lineages was 24\% (Table S3), which we interpret as a lack of statistical power due to the difference of 24 genera between the $75 \%$ and $90 \%$ majority-rule datasets. The equivalent BiSSE model with an extinction fraction equal in unisexual and bisexual lineages and different transition rates between unisexuality and bisexuality had a delta AIC of 24.4 and 27.2 as compared to the best-fit HiSSE model with the $75 \%$ and $90 \%$ majority-rule datasets, respectively. These results suggest that although sexual systems significantly influence diversification, other biological traits may also contribute to the observed diversification patterns.

The best-fit HiSSE model identified on the maximum clade credibility tree consistently returned a better fit than the 'nullfour' model on the 100 randomly sampled trees for the $75 \%$ and 90\% majority-rule datasets (Figs S1a, S2a), strengthening the result that diversification rates depend upon sexual condition in liverworts. When compared to a model with equal turnover rates, the model allowing different transition rates between unisexuality and bisexuality was still identified as the best-fit model over the 100 trees, although support for that model was weak with the $75 \%$ majority-rule dataset, pointing to some levels of phylogenetic uncertainty regarding the bias in transition rates. Taken together, most of the trees supported differential transition rates between unisexuality and bisexuality $(90 \%$ majority-rule dataset: $54 \%$ of the trees supported differential transition rates, $16 \%$ of the trees yielded nonsignificant results and 30\% favored other models; $75 \%$ majority-rule dataset: $83 \%$ of the trees supported differential transition rates, $7 \%$ returned nonsignificant results and $10 \%$ favoured other competing models) (see Figs S1b, S2b).

Net diversification rates measured on the chronogram under model 30 when sexual systems were scored with the modal 
condition of $75 \%$ were twice the magnitude in bisexual lineages (0.049) when compared to unisexual ones (0.024) (Table 1) and this trend was confirmed when the same model was applied to the 100 randomly sampled trees (Fig. S1d). Similar results were obtained when sexual systems were scored with the modal condition of $90 \%$, with higher net diversification rates measured on the chronogram in bisexual lineages (0.035) as compared to unisexual ones (0.022) (Table S4) and a clear bias towards higher net diversification rates in bisexual vs unisexual lineages across the 100 randomly sampled trees (Fig. S3d). The hidden states tended towards higher net diversification rates under state 1 than 0 (Tables S5, S6), suggesting that unmeasured traits also play a role in the observed patterns of diversification in liverworts.

Ancestral character state reconstructions (Figs 1, S3 for the $75 \%$ and $90 \%$ majority-rule datasets, respectively) revealed an overall pattern wherein transition rates were biased towards unisexuality (qdio-mono: 0.0018, qmono-dio: 0.016), and this trend was confirmed across the 100 randomly sampled trees (Figs S1, S2). In addition, unisexuality was identified as the most likely ancestral state of the liverworts with multiple subsequent independent transitions to bisexuality, ultimately followed by reversals to unisexuality, as best seen in complex thalloid species and Lejeuneaceae.

Sexual systems exhibited a strong and significant phylogenetic signal at the deepest nodes of the phylogeny but the signal decreased to become indistinguishable from the signal observed for randomized characters in the Lejeuneaceae and complex thalloids (Fig. 2).

\section{Discussion}

We found that bisexual lineages of liverworts have higher diversification rates than unisexual lineages, as expected under our primary hypothesis. This pattern is best exemplified by the markedly increased diversification in the bisexual lineage Lejeuneaceae, the most species-rich family of liverworts with a strong tropical presence. The finding that bisexuality is associated with significantly increased diversification strikingly contrasts with previous findings for angiosperms (Käfer et al., 2014). To explain these contrasting results, we suggest two possible mechanisms.

First, the faster diversification observed in bisexual liverworts is consistent with Heilbuth's (2000) hypothesis that random longdistance dispersals, possibly followed by allopatric speciation, are

Table 1 Speciation (lambda), extinction (mu) and net diversification (netdiv) rates in liverworts as inferred from the best-fit trait-dependent diversification model (Supporting Information Table S2) depending on the sexual condition (unisexual vs bisexual, as scored according to a majority rule fixed at $75 \%$ of the constitutive species of each genus) and an additional, unmeasured ('hidden') trait on the chronogram (Fig. 1)

\begin{tabular}{llll}
\hline & lambda & mu & netdiv \\
\hline Unisexual & 0.125 & 0.101 & 0.024 \\
Bisexual & 0.256 & 0.207 & 0.049 \\
Hidden 0 & 0.053 & 0.043 & 0.010 \\
Hidden 1 & 0.180 & 0.145 & 0.034 \\
\hline
\end{tabular}

more likely in bisexual lineages than in unisexual ones. Even though this hypothesis has been recently challenged by some evidence that specialized asexual diaspores may also contribute to long-distance dispersal across liverworts (Laenen et al., 2015), Heilbuth's hypothesis remains consistent with the observation that bisexual bryophytes are proportionally more common on oceanic islands than on continents, not because of the in situ evolution of bisexuality, but because bisexual species produce a greater number of sporophytic capsules (Patiño et al., 2013). Hence, larger numbers of spores are potentially able to reach oceanic islands owing to random long-distance dispersal, which is precisely the main driver of speciation in organisms, such as bryophytes, that typically fail to radiate and slowly diversify by genetic drift in allopatry (Patiño et al., 2014; Patiño \& Vanderpoorten, 2015).

Second, the apparently increased extinction of self-compatible species in angiosperms has been interpreted in terms of reduced genetic diversity and lower rates of recombination (Wright $\&$ Barrett, 2010), both inherent to bisexual bryophytes. Theoretical (Frankham, 1995; Lynch etal., 1995) and experimental (Nieminen et al., 2001) studies further demonstrate that inbreeding depression can cause high extinction risks due to the accumulation of slightly deleterious alleles in small populations. Although further research on inbreeding depression in bryophytes is necessary, some evidence suggests that the effects of bryophyte inbreeding are mitigated by the rapid purge of deleterious mutations during the gametophytic stage (Taylor et al., 2007; Jesson et al., 2011; Johnson \& Shaw, 2015). In particular, bisexual species are thought to rapidly purge recessive deleterious mutations through intra-gametophytic selfing (i.e. merging of gametes produced by shoots from the same protonema and hence, originating from the same spore (Taylor et al., 2007)). Such differences on the impact of inbreeding depression in bryophytes and angiosperms could also account for the observed difference in the association between diversification rates and sexual systems in the two groups.

Our phylogenetic reconstructions of sexual systems revealed that bisexuality has evolved within liverworts multiple times independently, from their unisexual ancestral condition. In bryophytes, chromosomal sex determination has been reported by several studies, which hypothesized that bisexuality is linked to polyploidy (Crawford et al., 2009). Indeed, if sex determination in bryophytes is chromosomal (reviewed in McDaniel \& Perroud, 2012), then the $X$ and $Y$ chromosomes in the sporophyte segregate at meiosis to give female gametophytes bearing an $\mathrm{X}$ and males bearing a $\mathrm{Y}$ chromosome. If polyploidy arises, then the genotype of the sporophyte generation would be XXYY. Depending on the pairing of $X$ and $Y$ chromosomes during meiosis, gametophytes could be XX, XY or YY. The XY gametophytes could thus result in the expression of both male and female sexual structures and, hence, lead to the evolution of bisexuality (Crawford et al., 2009). The evidence for simultaneous transitions in chromosome numbers and sexual systems has been, however, inconclusive in mosses (Crawford etal., 2009) and in liverworts. In fact, only c. 5\% of liverwort species are polyploids (Fritsch, 1991). This suggests that, if bisexuality and 


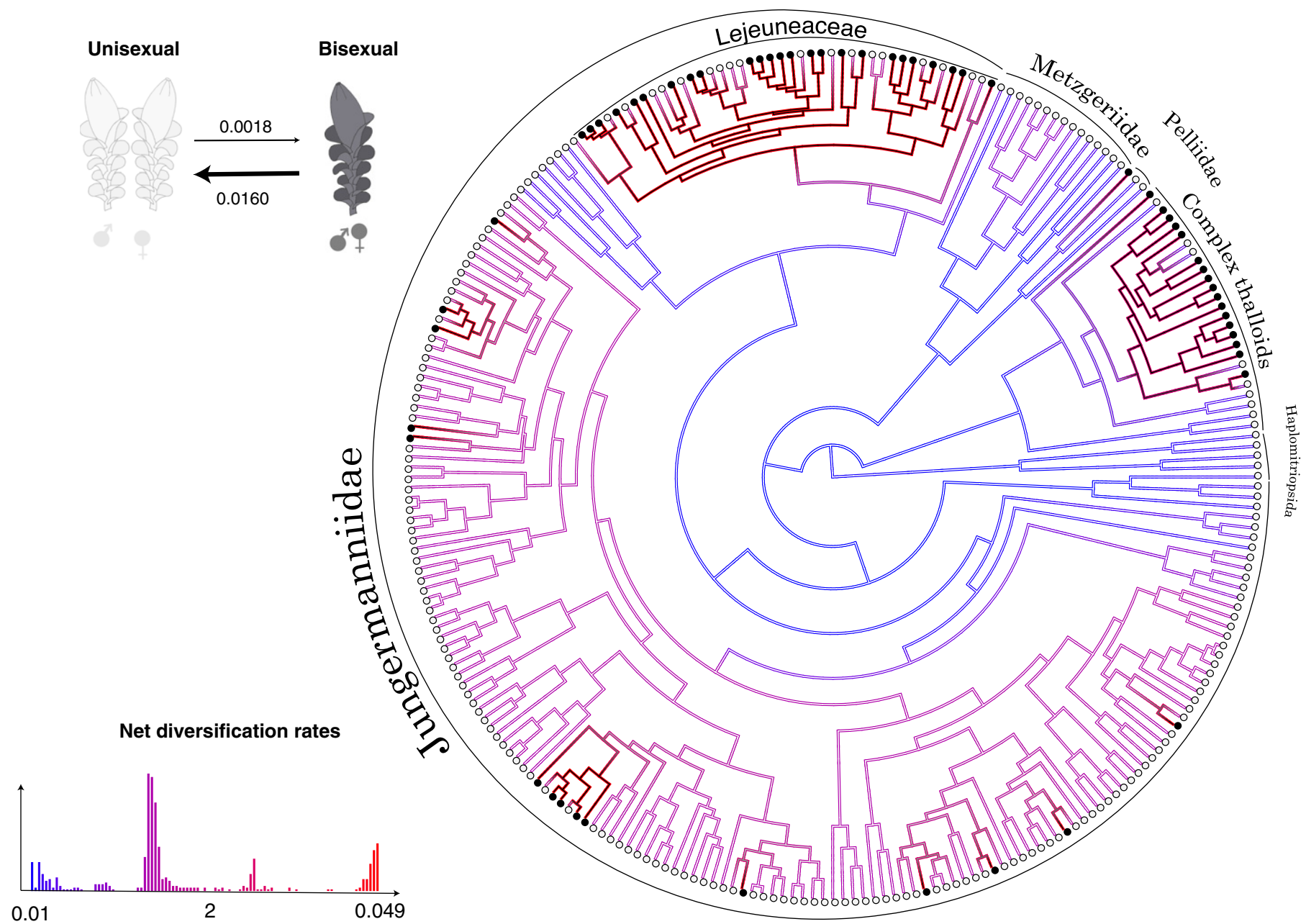

Fig. 1 Net diversification rates (increasing from blue to red) and shifts in sexual systems across liverworts using hidden state speciation and extinction (HiSSE; see the Materials and Methods section for details). The state at the tips represents the sexual condition observed in $>75 \%$ of the species constitutive of each genus. Ancestral estimation of the sexual systems is represented by black branches for bisexuals and white branches for unisexuals. The upper-left panel shows the transition rates between sexual systems and the bottom-left panel shows the distribution of net diversifications rates along the phylogeny.

polyploidization are associated, substantial asymmetrical gene and/or chromosomal loss would take place after the polyploidization. Ancient polyploidization and subsequent loss of many copies of duplicated genes has been recurrently reported for angiosperms (Paterson et al., 2004; Liu et al., 2014); an observation that calls for a thorough investigation of genome evolution among bryophytes. Nonetheless, the repeated evolution toward bisexuality in Lejeuneaceae and in the complex thalloid liverworts suggests that bisexuality might be a response to environmental conditions. The complex thalloid liverworts typically inhabit ephemeral or cyclically changing habitats, especially in climates with a harsh dry season, exhibit an annual life cycle and produce a few, large spores that maintain their germination capacity in the diaspore bank (Bischler, 2004; Kürschner \& Frey, 2013). In such harsh environments, bisexuality has a clear selective advantage because it increases the probability of fertilization and spore production as reported for desert mosses by Stark (1983). In Lejeuneaceae, the majority of species are epiphytic or epiphyllous, such that the bisexual condition and the associated production of sporophytes might be seen as an adaptive response to the selective pressure toward high dispersal in a cyclic environment.

Although our analyses indicate that some unmeasured traits may have contributed to liverwort diversification, suggesting the need for further research on these potentially relevant traits (e.g. growth form, symbiotic interactions, niche preference; see Bouchenak-Khelladi et al., 2015), bisexuality emerged as a significant factor and potentially a key innovation associated with higher diversification rates in liverworts. Yet, the ancestral unisexual condition still prevails across extant liverworts despite hundreds of millions of years of possible transitions toward bisexuality and despite the accelerated diversification of bisexual liverworts. The persistence of unisexuality resulted in a strong phylogenetic signal in sexual systems across the liverwort phylogeny, coupled with the highly phylogenetically structured distribution of bisexuality, mostly owing to the two major liverwort clades, the complex thalloids and the Lejeuneaceae, where bisexuality arose and has been largely maintained over time. These patterns contrast with the high lability of sexual systems in mosses 


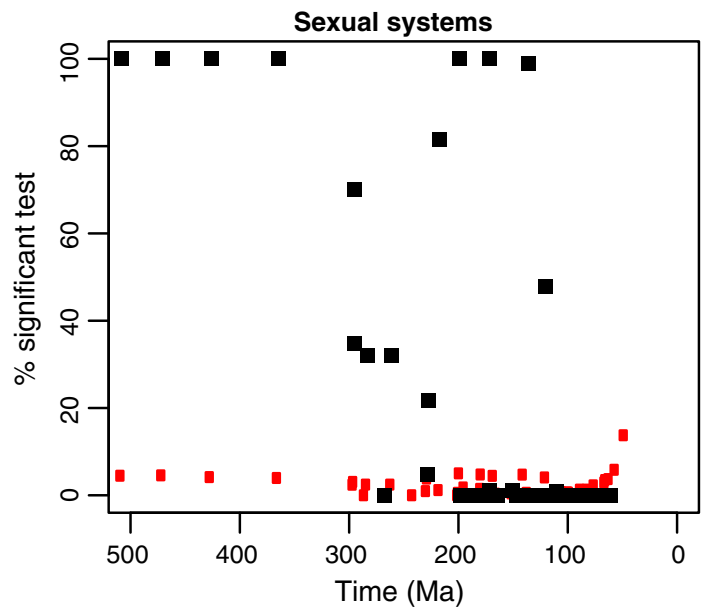

Fig. 2 Statistical significance of the phylogenetic signal in sexual systems in liverworts (as scored according to a majority rule fixed at $75 \%$ of the constitutive species of each genus) with respect to tree depth. The $y$-axis represents the proportion of trees, among 100 chronograms sampled at random from the posterior probability distribution of an analysis of 297 liverwort genera sequenced at eight loci and calibrated with 25 fossils, for which a significant phylogenetic signal ( $P$-value of the hierarchical likelihood ratio test between phylogenetic and nonphylogenetic models of trait evolution $<0.05$ ) was observed. Black dots represent actual data, whereas red dots represent the results obtained for randomly reshuffled characters (see the Materials and Methods section for details). Ma, Myr ago.

(McDaniel et al., 2013) and angiosperms (Weller \& Sakai, 1999; Renner, 2015) and we detected such lability only across the shallowest nodes of the liverwort phylogeny where the phylogenetic signal in sexual systems tended to decrease. The pattern of increasing lability of the sexual condition towards the tips of the tree is paralleled by the complete absence of phylogenetic signal in sexual systems within certain genera such as Radula (Devos et al., 2011) and Diplasiolejeunea (Dong et al., 2012). In other genera, however, the evolution of sexual systems seems to be much more phylogenetically constrained (e.g. Lejeunea; Heinrichs etal., 2013). Although the bisexual condition has been apparently stable and fixed in some lineages, it appears extremely scattered in others, as if it were the result of a trade-off between the advantages of self-compatibility, secured fertilization and sexual reproduction, on the one hand, and the evolutionary benefits of unisexuality, on the other.

The detected bias in transitions from bisexuality toward unisexuality observed in liverworts parallels the results previously reported in mosses (McDaniel et al., 2013). The bias towards unisexuality in both mosses and liverworts cannot be explained, as discussed above, in terms of high extinction rates associated with low genetic diversity due to selfing in bisexual lineages. Instead, it suggests that the unisexual condition confers some selective advantage over bisexuality, at least in these two groups. In unisexual species, male and female are sometimes completely allopatric. As an extreme example, only female plants of the moss Syntrichia pagorum occur in North America, whereas only male plants are known from Europe (Longton, 1997). In the liverwort Plagiochila exigua, only male plants occur in Europe whereas female plants occur in North America (Schuster, 1980; Longton
\& Schuster, 1983). The spatial segregation of males and females is reflected in their dramatically different biology. In particular, male and female gametophytes of unisexual species often exhibit strong sexual dimorphism, differences in size, growth rate, prezygotic reproductive investment (Stark \& McLetchie, 2006; Benassi etal., 2011; Horsley etal., 2011; Brzyski etal., 2014) and, possibly, in their ecological niche (Stark \& McLetchie, 2006; Brzyski et al., 2014; but see Bisang et al., 2015). The distinct distribution patterns and different life-history traits among sexes of unisexual species suggest that male and female plants basically behave as distinct species, which might represent a strong selective pressure for the maintenance of unisexuality.

\section{Acknowledgements}

This research was supported by a multi-institutional, collaborative grant from the Assembling the Tree of Life program at NSF (grant EF-0531730-002 to A.J.S.). B.L.'s work has been supported by the European Union (BeiPD-cofund Marie Curie) whereas A.V. is funded by the Belgian Funds for Scientific Research (FNRS). Many thanks are due to three referees for their constructive comments on the manuscript.

\section{Author contributions}

A.V. and B.L. designed the study. B.L. conducted the analyses. S.R.G. compiled the database. A.V. and B.L. wrote the manuscript with the help of A.M., B.S., J.P., A.D., B.G., C.J.C., S.R.G. and J.S.

\section{References}

Baker HG. 1955. Self-compatibility and establishment after 'long distance' dispersal. Evolution 9: 347-348.

Baker HG. 1967. Support for Baker's law as a rule. Evolution 21: 853-856.

Barrett SC. 2013. The evolution of plant reproductive systems: how often are transitions irreversible? Proceedings of the Royal Society B 280: 20130913.

Beaulieu JM, O'Meara BC. 2015. Detecting hidden diversification shifts in models of trait-dependent speciation and extinction. bioRxiv, in press. doi: http://dx.doi.org/10.1101/016386

Benassi M, Stark LR, Brinda JC, McLetchie DN, Bonine M, Mishler BD. 2011. Plant size, sex expression and sexual reproduction along an elevation gradient in a desert moss. The Bryologist 114: 277-288.

Bisang I, Ehrlén J, Korpelainen H, Hedenäs L. 2015. No evidence of sexual niche partitioning in a dioecious moss with rare sexual reproduction. Annals of Botany 116: 771-779.

Bisang I, Ehrlén J, Persson C, Hedenäs L. 2014. Family affiliation, sex ratio and sporophyte frequency in unisexual mosses. Botanical Journal of the Linnean Society 174: 163-172.

Bischler H. 2004. Liverworts of the Mediterranean: ecology, diversity, and distribution. Berlin-Stuttgart, Germany: J. Cramer.

Bouchenak-Khelladi Y, Onstein RE, Xing Y, Schwery O, Linder HP. 2015. On the complexity of triggering evolutionary radiations. New Phytologist 207: 313-326.

Brzyski JR, Taylor W, McLetchie DN. 2014. Reproductive allocation between the sexes, across natural and novel habitats, and its impact on genetic diversity. Evolutionary Ecology 28: 247-261.

Cheptou PO, Schoen DJ. 2007. Combining population genetics and demographical approaches in evolutionary studies of plant mating systems. Oikos 116: 271-279. 
Crawford M, Jesson LK, Garnock-Jones PJ. 2009. Correlated evolution of sexual system and life history traits in mosses. Evolution 63: 1129-1142.

Cusimano N, Renner SS. 2014. Ultrametric trees or phylograms for ancestral state reconstruction: does it matter? Taxon 63: 721-726.

De Vos JM, Hughes CE, Schneeweiss GM, Moore BR, Conti E. 2014. Heterostyly accelerates diversification via reduced extinction in Darwin's primroses. Proceedings of the Royal Society B 281: 20140075.

Devos N, Renner M, Gradstein SR, Shaw AJ, Laenen B, Vanderpoorten A. 2011. Evolution of sexual systems, dispersal strategies and habitat selection in the liverwort genus Radula. New Phytologist 192: 225-236.

Dong SS, Schäfer-Verwimp A, Meinecke P, Feldberg K, Bombosch A, Pócs T, Schmidt AR, Reitner J, Schneider H, Heinrichs J. 2012. Tramps, narrow endemics and morphologically cryptic species in the epiphyllous liverwort Diplasiolejeunea. Molecular Phylogenetics and Evolution 65: 582-594.

Donoghue MJ, Sanderson MJ. 2015. Confluence, synnovation, and depauperons in plant diversification. New Phytologist 207: 260-274.

Eppley SM, Taylor PJ, Jesson LK. 2007. Self-fertilization in mosses: a comparison of heterozygote deficiency between species with combined versus separate sexes. Heredity 98: 38-44.

FitzJohn RG. 2012. Diversitree: comparative phylogenetic tests of diversification. Methods in Ecology and Evolution 3: 1084-1092.

FitzJohn RG, Maddison WP, Otto SP. 2009. Estimating trait-dependent speciation and extinction rates from incompletely resolved phylogenies. Systematic Biology 58: 595-611.

Frankham R. 1995. Conservation genetics. Annual Review of Genetics 29: 305-327.

Fritsch R. 1991. Index to Bryophyte chromosome numbers. Berlin-Stuttgart, Germany: J. Cramer.

Goldberg EE, Kohn JR, Lande R, Robertson KA, Smith SA, Igić B. 2010. Species selection maintains self-incompatibility. Science 330: 493-495.

Harmon LJ, Weir JT, Brock CD, Glor RE, Challenger W. 2008. GEIGER: investigating evolutionary radiations. Bioinformatics 24: 129-131.

Heilbuth JC. 2000. Lower species richness in dioecious clades. The American Naturalist 156: 221-241.

Heilbuth JC, Ilves KL, Otto SP. 2001. The consequences of dioecy for seed dispersal: modeling the seed-shadow handicap. Evolution 55: 880-888.

Heinrichs J, Dong S, Schäfer-Verwimp A, Pócs T, Feldberg K, Czumaj A, Schmidt AR, Reitner J, Renner MAM, Hentschel J et al. 2013. Molecular phylogeny of the leafy liverwort Lejeunea (Porellales): evidence for a Neotropical origin, uneven distribution of sexual systems and insufficient taxonomy. PLoS One 8: e82547.

Horsley K, Stark LR, McLetchie DN. 2011. Does the silver moss Bryum argenteum exhibit sex-specific patterns in vegetative growth rate, asexual fitness or prezygotic reproductive investment? Annals of Botany 107: 897-907.

Hutsemékers V, Hardy OJ, Vanderpoorten A. 2013. Does water facilitate gene flow in spore-producing plants? Insights from the fine-scale genetic structure of the aquatic moss Rhynchostegium riparioides. Aquatic Botany 108: 1-6.

Jesson LK, Cavanagh AP, Perley DS. 2011. Polyploidy influences sexual system and mating patterns in the moss Atrichum undulatum sensu lato. Annals of Botany 107: 135-143.

Johnson MG, Shaw AJ. 2015. Genetic diversity, sexual condition, and microhabitat preference determine mating patterns in Sphagnum (Sphagnaceae) peat-mosses. Biological Journal of the Linnean Society 115: 96-113.

Käfer J, de Boer HJ, Mousset S, Kool A, Dufay M, Marais GA. 2014. Dioecy is associated with higher diversification rates in flowering plants. Journal of Evolutionary Biology 27: 1478-1490.

Käfer J, Mousset S. 2014. Standard sister clade comparison fails when testing derived character states. Systematic Biology 63: 601-609.

Klips RA. 2015. DNA microsatellite analysis of sporophytes of the short-lived arable moss Physcomitrium pyriforme reveals a predominantly self-fertilizing mating pattern. The Bryologist 118: 200-211.

Kürschner H, Frey W. 2013. Life strategies in bryophytes - a prime example for the evolution of functional types. Nova Hedwigia 96: 83-116.

Laenen B, Machac A, Gradstein SR, Shaw B, Patiño J, Désamoré A, Goffinet B, Cox CJ, Shaw AJ, Vanderpoorten A. 2015. Geographic range in liverworts: does sex really matter? Journal of Biogeography. doi: 10.1111/jbi.12661.
Laenen B, Shaw B, Schneider H, Goffinet B, Paradis E, Désamoré A, Heinrichs J, Villarreal JC, Gradstein SR, McDaniel SF et al. 2014. Extant diversity of bryophytes emerged from successive post-Mesozoic diversification bursts. Nature Communications 5: 6134.

Litsios G, Salamin N. 2012. Effects of phylogenetic signal on ancestral state reconstructions. Systematic Biology 61: 533-538.

Liu S, Liu Y, Yang X, Tong C, Edwards D, Parkin IAP, Zhao M, Ma J, Yu J, Huang S et al. 2014. The Brassica oleracea genome reveals the asymmetrical evolution of polyploid genomes. Nature Communications 5: e3930.

Longton RE. 1997. Reproductive biology and life-history strategies. Advances in Bryology 6: 65-101.

Longton RE, Schuster RM. 1983. Reproductive biology. In: Schuster RM, ed. New manual of bryology, vol. 1. Nichinan, Japan: Hattori Botanical Laboratory, 386-462.

Lynch M, Conery J, Bürger R. 1995. Mutation accumulation and the extinction of small populations. The American Naturalist 146: 489-518.

Machac A, Zrzavý J, Storch D. 2011. Range size heritability in Carnivora is driven by geographic constraints. The American Naturalist 177: 767-779.

Maddison WP, Midford PE, Otto SP. 2007. Estimating a binary character's effect on speciation and extinction. Systematic Biology 56: 701-710.

Maddison WP, FitzJohn RG. 2015. The unsolved challenge to phylogenetic correlation tests for categorical characters. Systematic Biology 64: 127-136.

McDaniel SF, Atwood J, Burleigh JG. 2013. Recurrent evolution of dioecy in bryophytes. Evolution 67: 567-572.

McDaniel SF, Perroud PF. 2012. Invited perspective: bryophytes as models for understanding the evolution of sexual systems. The Bryologist 115: 1-11.

McLetchie DN. 1996. Sperm limitation and genetic effects on fecundity in the dioecious liverwort Sphaerocarpos texanus. Sexual Plant Reproduction 9: 87-92.

Mooers AØ, Vamosi SM, Schluter D. 1999. Using phylogenies to test macroevolutionary hypotheses of trait evolution in Cranes (Gruinae). The American Naturalist 154: 249-259.

Nieminen M, Singer MC, Fortelius W, Schöps K, Hanski I. 2001. Experimental confirmation that inbreeding depression increases extinction risk in butterfly populations. American Naturalist 157: 237-244.

Oakley TH, Gu Z, Abouheif E, Patel NH, Li WH. 2005. Comparative methods for the analysis of gene-expression evolution: an example using yeast functional genomic data. Molecular Biology and Evolution 22: 40-50.

Pagel M, Meade A, Barker D. 2004. Bayesian estimation of ancestral character states on phylogenies. Systematic Biology 53: 673-684.

Paterson AH, Bowers JE, Chapman BA. 2004. Ancient polyploidization predating divergence of the cereals, and its consequences for comparative genomics. Proceedings of the National Academy of Sciences, USA 101: 99039908.

Patiño J, Bisang I, Hedenas L, Dirkse G, Bjarnason AH, Ah-Peng C, Vanderpoorten A. 2013. Baker's law and the island syndromes in bryophytes. Journal of Ecology 101: 1245-1255.

Patiño J, Carine MA, Fernández-Palacios JM, Otto R, Schaefer H, Vanderpoorten A. 2014. The anagenetic world of spore-producing land plants. New Phytologist 201: 305-311.

Patiño J, Vanderpoorten A. 2015. Macaronesia is a departure gate of anagenetic speciation in the moss genus Rhynchostegiella. Journal of Biogeography 42: 2122-2130.

Pellicer J, Kelly LK, Leitsch IJ, Zomlefer WB, Fay MF. 2014. A universe of dwarfs and giants: genome size and chromosome evolution in the monocot family Melanthiaceae. New Phytologist 201: 1484-1497.

Rabosky DL, Goldberg EE. 2015. Model inadequacy and mistaken inferences of trait-dependent speciation. Systematic Biology 64: 340-355.

Renner SS. 2015. The relative and absolute frequencies of angiosperm sexual systems: dioecy, monoecy, gynodioecy, and an updated online database. American Journal of Botany 101: 1588-1596.

Rydgren K, Cronberg N, Økland RH. 2006. Factors influencing reproductive success in the clonal moss, Hylocomium splendens. Oecologia 147: 445-454.

Schluter D, Price TD, Mooers AØ, Ludwig D. 1997. Likelihood of ancestor states in adaptive radiation. Evolution 51: 1699-1711.

Schuster RM. 1980. The hepaticae and anthocerotae of North America, vol. IV. New York, NY, USA: Columbia University Press. 
Schwery O, Onstein RE, Bouchenak-Khelladi Y, Xing Y, Carter RJ, Linder HP. 2015. As old as the mountains: the radiations of the Ericaceae. New Phytologist 207: 355-367.

Shortlidge EE, Rosenstiel TN, Eppley SM. 2012. Tolerance to environmental desiccation in moss sperm. New Phytologist 194: 741-750.

Stark LR. 1983. Bisexuality as an adaptation in desert mosses. American Midland Naturalist 110: 445-448.

Stark LR, Brinda JC. 2013. An experimental demonstration of rhizautoicy, selfincompatibility, and reproductive investment in Aloina bifrons (Pottiaceae). The Bryologist 116: 43-52.

Stark LR, McLetchie DN. 2006. Gender-specific heat-shock tolerance of hydrated leaves in the desert moss Syntrichia caninervis. Physiologia Plantarum 126: $187-195$.

Taylor PJ, Eppley SM, Jesson LK. 2007. Sporophytic inbreeding depression in mosses occurs in a species with separate sexes but not in a species with combined sexes. American Journal of Botany 94: 1853-1859.

Vamosi JC, Otto SP. 2002. When looks can kill: the evolution of sexually dimorphic floral display and the extinction of dioecious plants. Proceedings of the Royal Society of London B: Biological Sciences 269: 1187-1194.

Vamosi JC, Vamosi SM. 2004. The role of diversification in causing the correlates of dioecy. Evolution 58: 723-731.

Villarreal JC, Renner SS. 2013. Correlates of monoicy and dioicy in hornworts, the apparent sister group to vascular plants. BMC Evolutionary Biology 13: e329.

Weller SG, Sakai AK. 1999. Using phylogenetic approaches for the analysis of plant breeding system evolution. Annual Review of Ecology and Systematics 30: 167-199.

Wiens JJ. 1998. The accuracy of methods for coding and sampling higher-level taxa for phylogenetic analysis: A simulation study. Systematic Biology 47: 381397.

Wright SI, Barrett SCH. 2010. The long-term benefits of self-rejection. Science 330: 459-460.

\section{Supporting Information}

Additional supporting information may be found in the online version of this article.
Fig. S1 HiSSE results for 100 trees using the majority rule fixed at $75 \%$.

Fig. S2 HiSSE results for 100 trees using the majority rule fixed at $90 \%$.

Fig. S3 Liverwort mating system reconstruction under HiSSE model using the majority rule fixed at $90 \%$.

Table S1 Distribution of sexual systems among species in 297 liverwort genera

Table S2 Comparison of HiSSE models using the majority rule fixed at $75 \%$

Table S3 Comparison of HiSSE models using the majority rule fixed at $90 \%$

Table S4 Best-fit HiSSE model result using the majority rule fixed at $90 \%$

Table S5 Transition rates among states of sexual systems using the majority rule of $75 \%$

Table S6 Transition rates among states of sexual systems using the majority rule of $90 \%$

Please note: Wiley Blackwell are not responsible for the content or functionality of any supporting information supplied by the authors. Any queries (other than missing material) should be directed to the New Phytologist Central Office.

\section{About New Phytologist}

- New Phytologist is an electronic (online-only) journal owned by the New Phytologist Trust, a not-for-profit organization dedicated to the promotion of plant science, facilitating projects from symposia to free access for our Tansley reviews.

- Regular papers, Letters, Research reviews, Rapid reports and both Modelling/Theory and Methods papers are encouraged. We are committed to rapid processing, from online submission through to publication 'as ready' via Early View - our average time to decision is $<27$ days. There are no page or colour charges and a PDF version will be provided for each article.

- The journal is available online at Wiley Online Library. Visit www.newphytologist.com to search the articles and register for table of contents email alerts.

- If you have any questions, do get in touch with Central Office (np-centraloffice@lancaster.ac.uk) or, if it is more convenient, our USA Office (np-usaoffice@lancaster.ac.uk)

- For submission instructions, subscription and all the latest information visit www.newphytologist.com 